\title{
Generating Optimal Contiguous Evaluations for Expression DAGs
}

\author{
C. W. Kessler* Thomas Rauber ${ }^{\dagger}$ \\ Computer Science Department \\ Universität des Saarlandes \\ Postfach 151150 \\ 66041 Saarbrücken, Germany \\ +49-681-302-4130
}

FAX 49-681-302-4290

$\{$ kessler,rauber\}@cs.uni-sb.de

\begin{abstract}
We consider the NP-complete problem of generating contiguous evaluations for expression DAGs with a minimal number of registers. We present two algorithms that generate optimal contiguous evaluation for a given DAG. The first is a modification of a complete search algorithm that omits the generation of redundant evaluations. The second algorithm generates only the most promising evaluations by splitting the DAG into trees with import and export nodes and evaluating the trees with a modified labeling scheme. Experiments with randomly generated DAGs and large DAGs from real application programs confirm that the new algorithms generate optimal contiguous evaluations quite fast.
\end{abstract}

Key words: program optimization, basic block, expression DAG, contiguous evaluation, register allocation, code generation

\section{Introduction}

Register allocation is one of the most important problems in compiler optimization. Using fewer registers is important if the target machine has not enough registers to evaluate an expression without storing intermediate results in the main memory (spilling). This is especially important for vector processors that are often used in parallel computers. Vector processors usually have a small number

\footnotetext{
${ }^{*}$ Research partly supported by DFG, SFB 124, TP D4. Present address: FB 4 Informatik, Universität Trier, D-54286 Trier, Germany

†'supported by DFG, SFB 124, TP D4
} 
of vector registers (e.g., the CRAY vector computers have 8 vector register of $64 \times 64$ bit) or a register file that can be partitioned into a number of vector registers of a certain length (e.g., the vector acceleration units of the CM5 have register files of length $128 \times 32$ bit that can be partitioned into 1, 2, 4 or 8 vector registers, see [1]). A vector operation is evaluated by splitting it into stripes that have the length of the vector registers and computing the stripes one after another. If the register file is partitioned into a small number of vector registers, each of these can hold more elements and the vector operation to be evaluated is split into fewer stripes. This saves initialization costs and results in a faster computation [2].

Scientific programs often contain large basic blocks. Large basic blocks can also result from the application of compiler techniques like loop unrolling [3] and trace scheduling [4]. Therefore, it is important to derive register allocation techniques that cope with large basic blocks [5].

Among the numerous register allocation schemes, register allocation and spilling via graph coloring $[6,7]$ is generally accepted to yield good results. But register allocation via graph coloring uses a fixed evaluation order within a given basic block $B$. This is the evaluation order specified in the input program. Often there exists an evaluation order for $B$ that allows to use fewer registers. By using this order, the global register allocation generated via graph coloring could be improved.

The reordering of the operations within a basic block can be arranged by representing the basic block by a number of directed acyclic graphs (DAGs). An algorithm to build the DAGs for a given basic block can be found in [8]. A basic block is evaluated by evaluating the corresponding DAGs. For the evaluation of a DAG $G$ the following results are known:

(1) If $G$ is a tree, the algorithm of Sethi and Ullman [9] generates an optimal evaluation in linear time. (In this paper, optimal always means: uses as few registers as possible. Recomputations are not allowed.)

(2) The problem of generating an optimal evaluation for $G$ is NP-complete, if $G$ is not restricted [10].

In this paper, we restrict the attention to contiguous evaluations. Experiments with randomly generated DAGs and with DAGs that are derived from real programs show that for nearly all DAGs, there exist a contiguous evaluation that is optimal. This leads to an algorithm that computes an optimal contiguous evaluation for a given DAG in time $O\left(n \cdot 2^{d}\right)$ where $d$ is the number of decision nodes [11]. Decision nodes are binary nodes on paths from the root of the DAG to a node with more than one father.

This paper improves this simple $O\left(n \cdot 2^{d}\right)$ algorithm that performs a rather inefficient complete search, by identifying and eliminating redundant evaluations. It also presents a new algorithm that splits the given DAG into a number of trees with import and export nodes and evaluates the trees with a modified labeling scheme. Import and export nodes constitute the connection between the generated trees: when evaluating the tree, export nodes are nodes that remain into registers because they are used later by neighboring trees. On the other hand, import nodes need not to be loaded into a register because they have been left there by the evaluation of a neighboring tree. To find an optimal 
contiguous evaluation, the new algorithm considers all possibilities to split the given DAG into trees and selects the splitting that uses the fewest registers. Experiments with DAGs from real applications show that the number of generated evaluations is quite small even for large DAGs. Therefore, the running time of the algorithm remains reasonable.

After giving some basic definitions in Section 2, we describe in Section 3 how the running time of the algorithm from [11] can be reduced by generating each evaluation only once. In Section 4, we show how the running time can be further reduced by splitting the DAG in several trees with import and export nodes and applying a modified labeling scheme to the trees. Section 5 describes the splitting procedure, Section 6 presents the modified labeling scheme for trees with import and export nodes and proves that the generated evaluations are optimal. Section 7 shows the experimental results that confirm that the described method can be used in practice to generate optimal contiguous evaluations even for large DAGs.

\section{Evaluating DAGs}

\subsection{Expression DAGs}

We assume that we are generating code for a single processor machine with general-purpose registers $\mathcal{R}=\left\{R_{0}, R_{1}, R_{2}, \ldots\right\}$ and a countable sequence of memory locations. The arithmetic machine operations are three-address instructions of the following types:

$$
\begin{array}{ll}
R_{k} \leftarrow R_{i} \text { op } R_{j} & \text { binary operation, op } \in\{+,-, \times, \ldots\}, \\
R_{k} \leftarrow \text { op } R_{i} & \text { unary operation, } \\
R_{k} \leftarrow \operatorname{Load}(a) & \text { load register } k \text { with the value in memory location } a \\
\operatorname{Store}(a) \leftarrow R_{k} & \text { store the value in register } k \text { into memory location } a,
\end{array}
$$

In the following, we assume $i \neq j \neq k \neq i$, for $R_{k}, R_{i}, R_{j} \in \mathcal{R}$ to facilitate the description. Note that the following considerations are also applicable, if $k=i$ or $k=j$.

Each input program can be partitioned into a number of basic blocks.

A directed graph is a pair $G=(V, E)$, where $V$ is a finite set of nodes and $E \subseteq V \times V$ is a set of edges. In the following, $n=|V|$ always stands for the number of nodes in the graph. A node $w$ is called operand or son of a node $v$, if $(w, v) \in E$. $v$ is called result or father of $w$, i.e., the edge is directed from the son to the father. A node with no father is called a root of $G$. A node which has no sons is a leaf, otherwise it is an inner node. We call a node with two sons binary and a node with only one son unary. In the following, we suppose for simplicity that the DAGs contain only unary and binary inner nodes.

The outdegree outdeg $(w)$ is the number of edges leaving $w$, i.e., the number of its fathers.

The data dependencies in a basic block can be described by a directed acyclic graph (DAG). The leaves of the DAG are the variables and constants occurring as operands in the basic block; the inner nodes represent intermediate results. An example is given in Figure 1. 

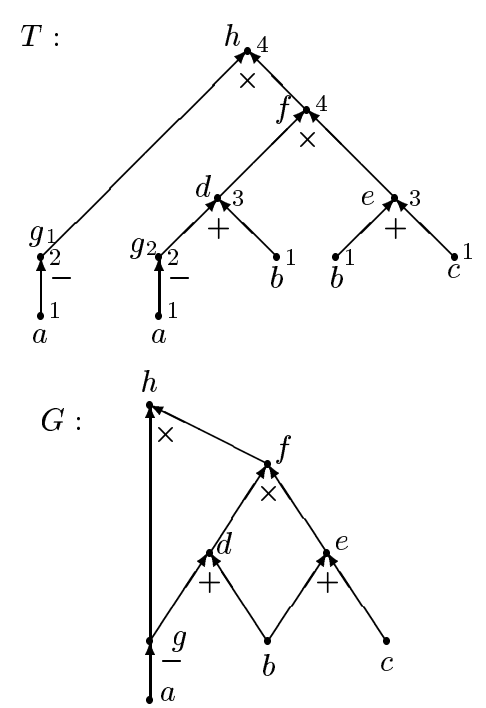

$R_{1} \leftarrow \operatorname{Load}(a)$

$R_{2} \leftarrow-R_{1}$

$R_{1} \leftarrow \operatorname{Load}(b)$

$R_{3} \leftarrow R_{2}+R_{1}$

$R_{1} \leftarrow \operatorname{Load}(b)$

$R_{2} \leftarrow \operatorname{Load}(c)$

$R_{4} \leftarrow R_{1}+R_{2}$

$R_{1} \leftarrow R_{3} \times R_{4}$

$R_{2} \leftarrow \operatorname{Load}(a)$

$R_{3} \leftarrow-R_{2}$

$R_{2} \leftarrow R_{3} \times R_{1}$

$A_{0}$ :

$\begin{array}{lll}R_{1} \leftarrow \operatorname{Load}(a) & c & R_{1} \leftarrow \operatorname{Load}(c) \\ R_{2} \leftarrow-R_{1} & b & R_{2} \leftarrow \operatorname{Load}(b) \\ R_{1} \leftarrow \operatorname{Load}(b) & e & R_{3} \leftarrow R_{1}+R_{2} \\ R_{3} \leftarrow R_{2}+R_{1} & a & R_{1} \leftarrow \operatorname{Load}(a) \\ R_{4} \leftarrow \operatorname{Load}(c) & g & R_{4} \leftarrow-R_{1} \\ R_{5} \leftarrow R_{1}+R_{4} & d & R_{1} \leftarrow R_{4}+R_{2} \\ R_{1} \leftarrow R_{3} \times R_{5} & f & R_{2} \leftarrow R_{1} \times R_{3} \\ R_{3} \leftarrow R_{2} \times R_{1} & h & R_{1} \leftarrow R_{4} \times R_{2}\end{array}$

Figure 1: Example: The expression $(-a) \times((-a+b) \times(b+c))$ can be represented by the tree $T$ shown above. The tree can be evaluated by the labeling algorithm of Sethi/Ullman with 4 registers as shown to the right. (The labels are printed at the right-hand side of the nodes). By eliminating common subexpressions, a DAG $G$ can be constructed. Evaluating $G$ in the original order results in an evaluation $A_{0}$ that reduces the number of instructions and hence the computation time of the basic block, but uses 5 instead of 4 registers. By reordering $A_{0}$ as shown to the right, we get an evaluation that needs only 4 registers.

Definition 1 (subDAG) Let $G=(V, E)$ be a DAG. A DAG $S=\left(V^{\prime}, E^{\prime}\right)$ is called subDAG of $G$, if $V^{\prime} \subseteq V$ and $E^{\prime} \subseteq E \cap\left(V^{\prime} \times V^{\prime}\right)$. A subDAG $S=\left(V^{\prime}, E^{\prime}\right)$ of $G=(V, E)$ with root $w$ is called complete, if:

$V^{\prime}=\{v \in V: \exists$ path from $v$ to $w\}$ and

$E^{\prime}=\left\{e \in E:\right.$ e is an edge on a path from a node $v \in V^{\prime}$ to $\left.w\right\}$.

\subsection{DAG Evaluations}

We now consider the evaluation of DAGs. Let $G=(V, E)$ be a directed graph with $n$ nodes. A mapping ord: $V \rightarrow\{1,2, \ldots, n\}$ with

$$
\forall(w, v) \in E: \quad \operatorname{ord}(w)<\operatorname{ord}(v)
$$

is called a topologic order of the nodes of $G$. It is well-known that for a directed graph $G$ a topological order exists iff $G$ is acyclic (e. g., [12]).

Definition 2 (evaluation of a DAG) An evaluation $A$ of a DAGG is a permutation of the nodes in $V$ such that for all nodes $v \in V$ the following holds: If $v$ is an inner node with sons $v_{1}, \ldots, v_{k}$, then $v$ occurs in $A$ behind $v_{i}, i=1, \ldots, k$.

This implies that the evaluation $A$ is complete and contains no recomputations, i. e., each node of the DAG appears exactly once in A. Moreover, the evaluation is consistent, because no node is evaluated 
before all of its sons are evaluated. Thus, each topological order of $G$ represents an evaluation, and vice versa.

Definition 3 (contiguous evaluation) An evaluation A (represented by the topological order ord) of a DAG $G=(V, E)$ is called contiguous, if for each node $v \in V$ with children $v_{1}$ and $v_{2}$ the following is true: if $w_{i}$ is a predecessor of $v_{i}, i=1,2$, and $\operatorname{ord}\left(v_{1}\right)<\operatorname{ord}\left(v_{2}\right)$, then $\operatorname{ord}\left(w_{1}\right)<\operatorname{ord}\left(w_{2}\right)$.

A contiguous evaluation of a node $v$ first evaluates the complete subDAG with one of the children of $v$ as root before evaluating any part of the remaining subDAG with root $v$.

While general evaluations can be generated by variants of topological-sort ${ }^{1}$, contiguous evaluations are generated by variants of $d e p t h-f i r s t$ search ( $d f s$ ). From now on, we restrict our attention to contiguous evaluations to reduce the number of generated evaluations. By doing so, we may not always get the evaluation with the least register need. There are some DAGs for which a general evaluation exists that uses fewer registers than every contiguous evaluation. However, these DAGs are usually quite large and do very rarely occur in real programs. The smallest DAG of this kind that we could construct so far has 14 nodes and is given in Figure 2. Note that for larger DAGs, it is quite difficult to decide whether there exists a general evaluation that uses fewer registers than every contiguous evaluation. This is because of the enormous running time of the algorithms that generate general evaluations.

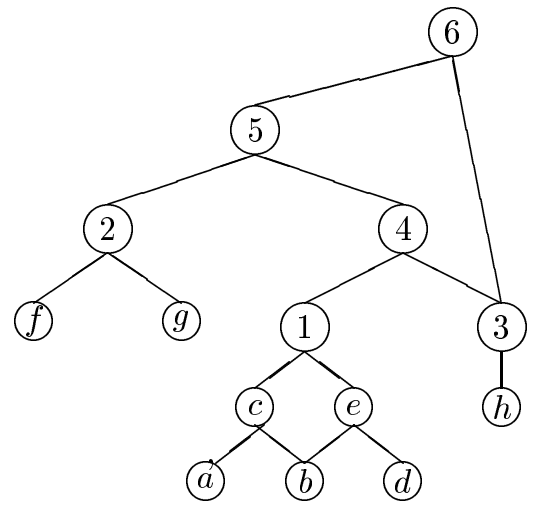

Figure 2: For this DAG, the noncontiguous evaluation $(a, b, c, d, e, 1, f, g, 2, h, 3,4,5,6)$ uses 4 registers. There is no contiguous evaluation that uses fewer than 5 registers. To use 4 registers, a contiguous evaluation would have to evaluate the subDAG with root 1 first. A contiguous evaluation could do this only by first evaluating the left son of node 6 , then the right son of node 5 and then the left son of node 4. In order this evaluation to be contiguous, nodes $h$ and 3 must be evaluated after 1 and thus, the values of nodes 3 and 4 must be held in two registers. But in order to evaluate nodes $f, g$ and 2 , three more registers would be required. Thus, the contiguous evaluation would use at least five registers altogether.

Definition 4 (register allocation, register need, optimal evaluation) (cf. [10]) Let num : $\mathcal{R} \rightarrow$ $\{0,1,2 \ldots\}, \operatorname{num}\left(R_{i}\right)=i$ be a function that assigns a number to each register. A mapping reg : $V \rightarrow \mathcal{R}$ is called a (consistent) register allocation for $A$, if for all nodes $u, v, w \in V$ the following holds: If $u$ is a son of $w$, and $v$ appears in $A$ between $u$ and $w$, then $\operatorname{reg}(u) \neq \operatorname{reg}(v)$.

$$
m(A)=\underset{\text { reg is reg. alloc. for } A}{\min }\left\{\max _{v \text { appears in } A}\{n u m(\operatorname{reg}(v))+1\}\right\}
$$

\footnotetext{
${ }^{1}$ See [12] for a summary on topological sorting.
} 
is called the register need of the evaluation A. An evaluation A for a DAGG is called optimal if for all evaluations $A^{\prime}$ of $G$ holds $m\left(A^{\prime}\right) \geq m(A)$.

Sethi proved in 1975 [10] that the problem of computing an optimal evaluation for a given DAG is NP-complete. Assuming $\mathbf{P} \neq \mathbf{N P}$, we expect an optimal algorithm to require nonpolynomial time.

\section{Counting Evaluations}

In [11], we give the following definitions and prove the following lemmata:

Definition 5 (tree node)

(1) Each leaf is a tree node.

(2) An inner node is a tree node iff all its sons are tree nodes and none of them has outdegree $>1$.

Definition 6 (label)

(1) For every leaf $v$, label $(v)=1$.

(2) For a unary node $v$, label $(v)=\max \{\operatorname{label}(\operatorname{son}(v)), 2\}$.

(3) For a binary node $v, \operatorname{label}(v)=\max \{3, \max \{\operatorname{label}(\operatorname{lson}(v)), \operatorname{label}(\operatorname{rson}(v))\}+q\}$

where $q=1$, if label $(\operatorname{lson}(v))=\operatorname{label}(\operatorname{rson}(v))$, and 0 otherwise.

Let new_reg() be a function that returns an available register and marks it to be busy. Let regfree(reg) be a function that marks the register reg to be free again. A possible implementation is given in Section 8. The Labeling-algorithm labelfs of Sethi and Ullman (see [9]) generates optimal evaluations for a tree with labels by first evaluating the son with the greater label value for each binary node.

(1) function labelfs (node $v)$

// generates an optimal evaluation for the subtree with root $v / /$

(2) if $v$ is not a leaf

(3) then if label $(\operatorname{lson}(v))>\operatorname{label}(\operatorname{rson}(v))$

(4) then labelfs $(\operatorname{lson}(v)) ; \operatorname{labelfs}(\operatorname{rson}(v))$

(5) $\quad$ else labelfs $(r s o n(v))$; labelfs $(\operatorname{lson}(v))$

fi

fi

(6) $\quad r e g(v) \leftarrow n e w \_r e g() ; \operatorname{print}(v, r e g(v))$;

$(7)$ if $v$ is not a leaf then regfree $(\operatorname{reg}(\operatorname{lson}(v)))$; regfree $(\operatorname{reg}(\operatorname{rson}(v))) \mathbf{f i}$

end labelfs; 


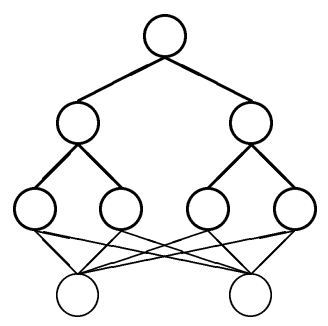

Figure 3: A DAG with $n-2$ decision nodes.

Definition 7 (decision node) $A$ decision node is a binary node which is not a tree node.

Thus, all binary nodes that have at least one predecessor with more than one father are decision nodes. In a tree, there are no decision nodes. For a general DAG let $d$ be the number of decision nodes and $b$ be the number of binary tree nodes. Then $k=b+d$ is the number of binary nodes of the DAG. A DAG may have up to $d=n-2$ decision nodes, see Figure 3.

Lemma 1 For a tree $T$ with one root and b binary nodes, there exist exactly $2^{b}$ different contiguous evaluations.

Lemma 2 For a DAG with one root and $k$ binary nodes, there exist at most $2^{k}$ different contiguous evaluations.

Lemma 3 Let $G$ be a DAG with $d$ decision nodes and b binary tree nodes which form $t$ (disjoint) subtrees $T_{1}, \ldots, T_{t}$. Let $b_{i}$ be the number of binary tree nodes in $T_{i}, i=1 \ldots t$, with $\sum_{i=1}^{t} b_{i}=b$. Then the following is true: If we fix an evaluation $A_{i}$ for $T_{i}$, then there remain at most $2^{d}$ different contiguous evaluations for $G$.

Corollary 4 If we evaluate all the tree nodes in a DAG $G$ with $d$ decision nodes by labelfs(), there remain at most $2^{d}$ different contiguous evaluations for $G$.

The following simple algorithm performs a complete search to create all $2^{d}$ contiguous evaluations for $G$, provided that a fixed contiguous evaluation for the tree nodes of $G$ is used:

(1) algorithm complete_search

(2) Let $v_{1}, \ldots, v_{d}$ be the decision nodes of a DAG $G$, and

(3) let $\beta=\left(\beta_{1}, \ldots, \beta_{d}\right) \in\{0,1\}^{d}$ be a bitvector.

(4) forall $2^{d}$ different $\beta \in\{0,1\}^{d}$ do

(5) $\quad$ start $d f s($ root) with each $\beta$, such that for $1 \leq i \leq d$

(6) if $\beta_{i}=0$ in the call $d f s\left(v_{i}\right)$,

(7) then the left son of $v_{i}$ is evaluated first

(8) else the right son of $v_{i}$ is evaluated first $\mathbf{f i}$

(9) od

end complete_search; 


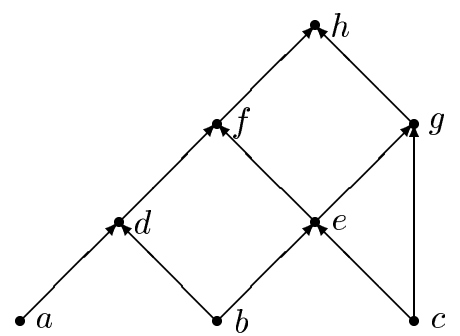

Figure 4: Example DAG.

This algorithm has exponential running time, since a DAG with $n$ nodes can have up to $d=n-2$ decision nodes, see Figure 3. The running time of the algorithm can be reduced by exploiting the following observation (consider the example DAG in Figure 4): Assume that the algorithm to generate a contiguous evaluation decides to evaluate the left son $f$ of the root $h$ first (i.e., the decision bit of $h$ is set to zero). Then node $e$ appears in the evaluation before $g$, since $e$ is in the subDAG of $f$, but $g$ is not. Therefore, there is no real decision necessary when node $g$ is evaluated, because the son $e$ of $g$ is already evaluated. But because $g$ is a decision node, the algorithm generates bitvectors containing 0s and 1 s for the decision bit of $g$, although bitvectors that only differ in the decision bit for $g$ describe the same evaluation.

We say that $g$ is excluded from the decision by setting the decision bit of $h$ to 0 , because the son $e$ (and $c$ ) are already evaluated when the evaluation of $g$ starts. We call the decision bit of $g$ redundant and mark it by an asterisk $(*)$.

The following algorithm computes only those bitvectors that yield different evaluations. We suppose again that tree nodes are evaluated by the labeling algorithm labelfs:

Let $v_{1}, \ldots, v_{d}$ be the decision nodes in reverse topological order (i.e., the root comes first)

We call the following function $\operatorname{descend}(\Theta, 1)$ where $\Theta$ is a bitvector that contains $d$ 0's.

(1) function descend ( bitvector $\beta$, int pos )

(2) while $\beta_{p o s}=*$ and $p o s<d$ do $p o s \leftarrow p o s+1$ od

(3) if $p o s \geq d$

(4) then if $\beta_{p o s}=*$

(5) then print $\beta$ // new evaluation found //

(6) else // $\beta_{\text {pos }}$ is empty: //

(9) else $\beta_{\text {pos }}=0$;

(10) mark exclusions of nodes $v_{j}, j \in\{$ pos $+1, \ldots, d\}$ through $l s o n\left(v_{\text {pos }}\right)$ by $\beta_{j} \leftarrow *$;

(11) descend $(\beta$, pos +1$)$;

(12) $\quad \beta_{\text {pos }}=1$;

(13) $\quad$ mark exclusions of nodes $v_{j}, j \in\{$ pos $+1, \ldots, d\}$ through $\operatorname{rson}\left(v_{\text {pos }}\right)$ by $\beta_{j} \leftarrow *$;

(14) $\quad$ descend $(\beta$, pos +1$)$; 


\begin{tabular}{|l|lllll|l|}
\hline decision nodes $v_{1}, v_{2}, \ldots, v_{5}:$ & $h$ & $f$ & $g$ & $d$ & $e$ & \\
\hline \hline start at the root: preset first bit: & $\mathbf{0}$ & & $*$ & & & \\
\hline propagate bits and asterisks to next stage: & 0 & $\mathbf{0}$ & $*$ & & $*$ & \\
\hline all bits set: first evaluation found: & 0 & 0 & $*$ & $\mathbf{0}$ & $*$ & $A_{1}$ \\
& 0 & 0 & $*$ & $\mathbf{1}$ & $*$ & $A_{2}$ \\
\hline 'backtrack': & 0 & $\mathbf{1}$ & $*$ & $*$ & & \\
\hline & 0 & 1 & $*$ & $*$ & $\mathbf{0}$ & $A_{3}$ \\
& 0 & 1 & $*$ & $*$ & $\mathbf{1}$ & $A_{4}$ \\
\hline 'backtrack': & $\mathbf{1}$ & $*$ & & $*$ & & \\
\hline & 1 & $*$ & $\mathbf{0}$ & $*$ & & \\
\hline & 1 & $*$ & 0 & $*$ & $\mathbf{0}$ & $A_{5}$ \\
& 1 & $*$ & 0 & $*$ & $\mathbf{1}$ & $A_{6}$ \\
\hline 'backtrack': & 1 & $*$ & $\mathbf{1}$ & $*$ & $*$ & $A_{7}$ \\
\hline \hline
\end{tabular}

Table 1: For the example DAG of Figure 4, the algorithm descend executes the above evaluation steps. Only 7 instead of $2^{5}=32$ contiguous evaluations are generated.

\section{fi \\ end descend;}

Table 1 shows the application of descend to the example DAG of Figure 4.

Lemma 5 For a DAGG without unary nodes, the algorithm descend generates at most $2^{d-1}$ different contiguous evaluations.

Proof: If there are only binary inner nodes, there must exist a DAG node $v$ that has at least two different fathers $w_{1}$ and $w_{2}$. Suppose $w_{1}$ is evaluated first. Then the decision bit of $w_{2}$ is redundant and is set to $*$. $\square$ Let $N$ be the number of different contiguous evaluations returned by the algorithm descend. We have $N=7$ for the example DAG of Figure 4 . We call $d_{\text {eff }}=\log N$ the effective number of decision nodes of $G$. It is $d_{\text {eff }} \leq d-1$ because of Lemma 3 and Lemma 6 .

Furthermore, we can show the following lower bound:

Lemma $6 \quad d_{\text {eff }} \geq_{P \text { path from the root to some leaf }}$ \#decision nodes on $P$

Proof: There must be at least as many bits set to 0 or 1 in each final bitvector as there are decision nodes on an arbitrary path from some leaf to the root, because no exclusion is possible on the path to the node being evaluated first. The bitvector describing the path with the smallest number of decision nodes is enumerated by the algorithm, so the lower bound follows. 

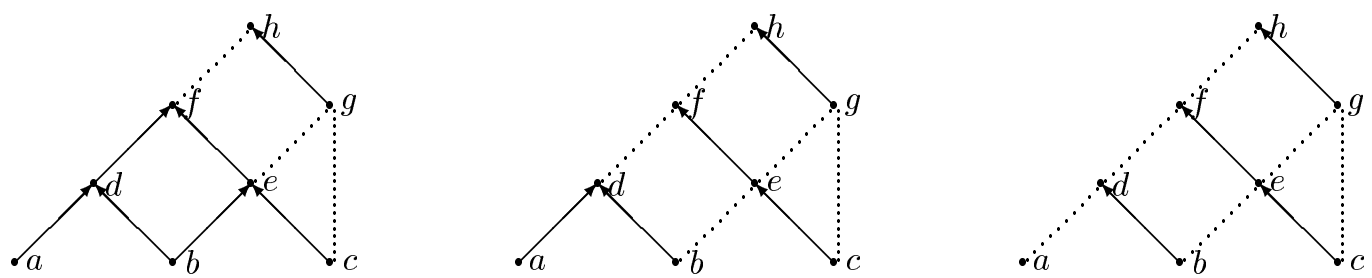

Figure 5: The example DAG is split in three steps by setting $\beta_{1}=0, \beta_{2}=0, \beta_{4}=0$. The edges between the generated subtrees are shown as dotted lines.

In the example above, the lower bound for $d_{e f f}$ is 2 , since the path with the least number of decision nodes is $(h, g, c)$ which has two decision nodes.

This lower bound may be used to get a lower bound $\left(2^{d}\right.$ eff $)$ for the run time of the algorithm descend.

\section{Reducing the Number of Evaluations}

We now construct an algorithm that reduces the number of generated evaluations further. The reduction is based on the following observation: Let $v$ be a decision node with two children $v_{1}$ and $v_{2}$. Let $G(v)=(V(v), E(v))$ be a DAG with root $v, G\left(v_{i}\right)$ the complete subDAG with root $v_{i}, i=1,2$. By deciding to evaluate $v_{1}$ before $v_{2}$, we decide to evaluate all nodes of $G\left(v_{1}\right)$ before the nodes in $G_{\text {rest }}=\left(V_{\text {rest }}, E_{\text {rest }}\right)$ with $V_{\text {rest }}=V(v)-V\left(v_{1}\right), E_{\text {rest }}=E(v) \cap\left(V_{\text {rest }} \times V_{\text {rest }}\right)$. Let $e=(u, w) \in E(v)$ be an edge with $u \in V\left(v_{1}\right), w \in V_{\text {rest }}$. The function descend marks $w$ with a $*$. This can be considered as eliminating $e$ : at decision node $w$, we do not have the choice to evaluate the son $u$ first, because $u$ has already been evaluated and will be held in a register until $w$ is evaluated. Therefore, descend can be considered as splitting the DAG $G$ into smaller subDAGs. We will see later that these subDAGs are trees after the splitting has been completed. The root of each of these trees is a decision node. ${ }^{2}$ The trees are evaluated in reverse of the order in which they are generated. For the example DAG of Figure 4, there are 7 possible ways of carrying out the splitting. The splitting steps that correspond to evaluation $A_{1}$ from Table 1 are shown in Figure 5.

If we look at the subDAGs that are generated during the splitting operation, we observe that even some of the intermediate subDAGs are trees which could be evaluated without a further splitting. E.g., after the second splitting step $\left(\beta_{2}=0\right)$ in Figure 5 , there is a subtree with nodes $a, b, d$ which does not need to be split further, because an optimal contiguous evaluation for the subtree can be found by a variant of labelfs.. By stopping the splitting operations in these cases, the number of generated evaluations can be reduced from 7 to 3 for the example DAG.

Depending on the structure of the DAG, the number of generated evaluations may be reduced dramatically when splitting the DAG into trees. An example is given in Figure 6. To evaluate the generated trees we need a modified labeling algorithm that is able to cope with the fact that some nodes of

\footnotetext{
${ }^{2}$ As we will see later, the root of the last generated tree is not a decision node.
} 

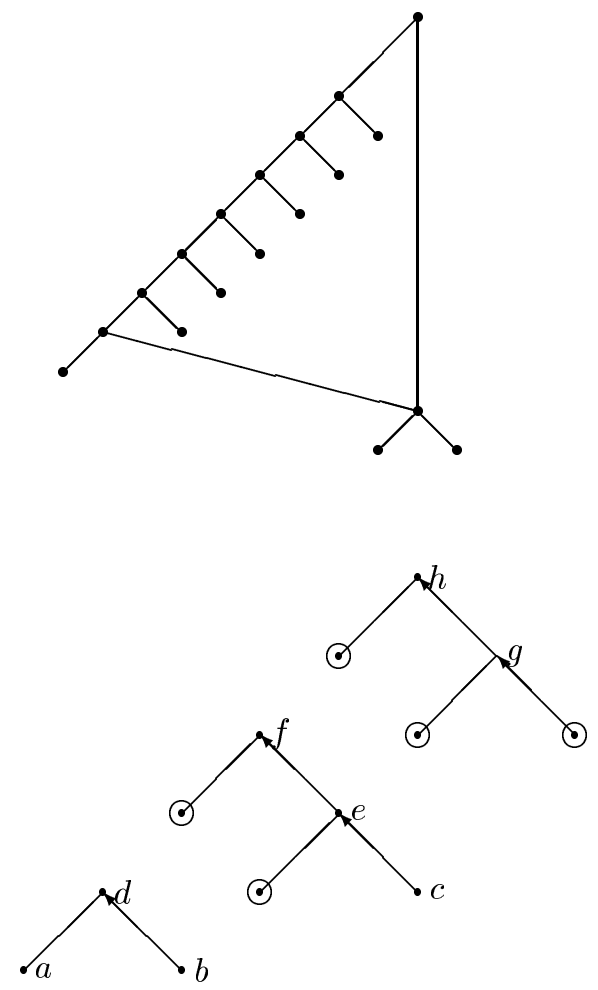

Figure 6: The DAG to the left has 8 decision nodes. When using the function descend, only one node gets an asterisk, i.e. $2^{7}$ evaluations are generated. When using the labeling version, only 2 evaluations are generated: the first one evaluates the left son of the root first, the second one evaluates the right son first.

Figure 7: The example DAG is split into 3 subtrees by setting $\beta_{1}=0, \beta_{2}=0, \beta_{4}=0$. The newly introduced import nodes are marked with a circle. They are all nonpermanent.

the trees must be held in a register until the last reference from any other tree is resolved. Such an algorithm is given in Section 6. Before applying the new labeling algorithm, we explicitly split the DAG in subtrees $T_{1}=\left(V_{1}, E_{1}\right), \ldots, T_{k}=\left(V_{k}, E_{k}\right)$. We suppose that these subtrees must be evaluated in this order. The splitting procedure is described in detail in the next section. After the splitting, we introduce additional import nodes which establish the communication between the trees. The resulting trees to the second DAG in Figure 5 are given in Figure 7.

We present the labeling algorithm in Section 6 with the notion of import and export nodes: An export node of a tree $T_{i}$ is a node which has to be left in a register because another tree $T_{j}(j>i)$ has a reference to $v$, i.e., $T_{j}$ has an import node which corresponds to $v$. An import node of $T_{i}$ is a leaf which is already in a register $R$ because another tree $T_{j}(j<i)$ that has been evaluated earlier has left the corresponding export node in $R$. Therefore, an import node need not to be loaded in a register and does not appear again in the evaluation. For each import node, there exists a corresponding export node. Two import nodes $v_{1} \neq v_{2}$ may have the same corresponding export node.

We distinguish two types of import nodes:

- A permanent input node $v$ can be evaluated without being loaded in a register. $v$ can not be removed from the register after the father of $v$ is evaluated, because there is another import node of $T_{i}$ or of another tree $T_{j}$ that has the same corresponding export node as $v$ and that has not been evaluated yet.

- A non-permanent input node $v$ can also be evaluated without being loaded into a register. But the register that contains $v$ can be freed after the father of $v$ has been evaluated, because all 
other import nodes that have the same corresponding export node as $v$ are already evaluated. ${ }^{3}$

Let the DAG nodes be $V=V_{1} \cup \ldots \cup V_{k}$. We describe the import and export nodes by the following characteristic functions:

$$
\begin{aligned}
& \exp : V \rightarrow\{0,1\} \quad \text { with } \exp (v)= \begin{cases}1 & \text { if } v \text { is an export node } \\
0 & \text { otherwise }\end{cases} \\
& i m p_{p}: V \rightarrow\{0,1\} \quad \text { with } \quad i m p_{p}(v)= \begin{cases}1 & \text { if } v \text { is a permanent import node } \\
0 & \text { otherwise }\end{cases} \\
& \operatorname{imp} p_{n p}: V \rightarrow\{0,1\} \quad \text { with } \quad i m p_{n p}(v)= \begin{cases}1 & \text { if } v \text { is a non-permanent import node } \\
0 & \text { otherwise }\end{cases} \\
& \operatorname{corr}: V \rightarrow V \quad \text { with } \operatorname{corr}(v)=u, \text { if } u \text { is the corresponding export node to } v
\end{aligned}
$$

The definition of import and export nodes implies

$$
\exp (v)+i m p_{p}(v)+i m p_{n p}(v) \leq 1 \text { for each } v \in V_{i}
$$

\section{Splitting the DAG into subtrees}

We now describe how the DAGs are split into subtrees and how the import and export nodes are determined. We derive a recursive procedure descend2 that is a modification of descend. descend2 generates a number of evaluations for a given DAG $G$ by splitting $G$ into subtrees and evaluating the subtrees with a modified labeling scheme. Among the generated evaluations are all optimal evaluations. We first describe how the splitting is executed.

Let $d$ be the number of decision nodes. The given DAG is split into at most $d$ subtrees to generate an evaluation. After each split operation, export nodes are determined and corresponding import nodes are introduced as follows: Let $v=v_{\text {pos }}$ be a decision node with children $v_{1}$ and $v_{2}$ and let $G(v), G\left(v_{1}\right)$ and $G_{r e s t}$ be defined as in the previous section. We consider the case that $v_{1}$ is evaluated before $v_{2}$ $\left(\beta_{\text {pos }}=0\right)$. Let $u \in V\left(v_{1}\right)$ be a node for which an edge $(u, w) \in E(v)$ with $w \in V_{\text {rest }}$ exists. Then $u$ is an export node in $G\left(v_{1}\right)$. A new import node $u^{\prime}$ is added to $G_{\text {rest }}$ by setting $V_{\text {rest }}=V_{\text {rest }} \cup\left\{u^{\prime}\right\}$ and $E_{\text {rest }}=E_{\text {rest }} \cup\left\{\left(u^{\prime}, w\right)\right\} . u^{\prime}$ is the corresponding import node to $u$. If $u$ has already been marked in $G\left(v_{1}\right)$ as export node, then $u^{\prime}$ is a permanent import node, because there is another reference to $u$ (from another tree) that is evaluated later. Otherwise, $u^{\prime}$ is a non-permanent import node. If there are other edges $e_{i}=\left(u, w_{i}\right) \in E(v)$ with $i=1, \ldots, k$ and $w_{i} \in V_{\text {rest }}$, then new edges $e_{i}^{\prime}=\left(u^{\prime}, w_{i}\right)$ are added to $E_{\text {rest }}$. If $k \geq 1, G_{\text {rest }}$ is not a tree and will be split later on.

A difficulty arises if $u=v_{1}$ is a leaf in $G(v)$ and there is a node $w \neq v$ in $V_{\text {rest }}$ with $e=\left(v_{1}, w\right) \in E(v)$, see Figure 8. Then $G\left(v_{1}\right)=\left(\left\{v_{1}\right\}, \emptyset\right)$. $w$ is a decision node that gets a $*, v$ is a decision node for

\footnotetext{
${ }^{3}$ This partitioning of the import nodes is well defined, since the order of the $T_{i}$ is fixed.
} 

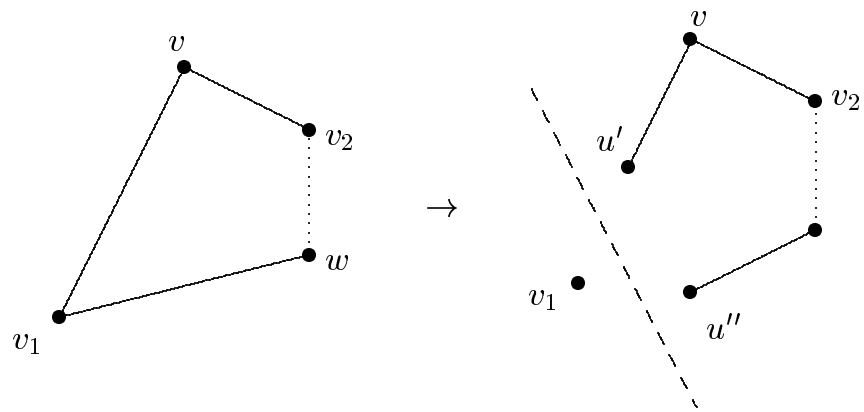

Figure 8: $v$ has two children $v_{1}$ and $v_{2} . v_{1}$ is a leaf, $w$ is a predecessor of $v_{2}$.

which the decision has been chosen. If $G_{\text {rest }}$ contains no other decision node except $v$ and $w$, we have the situation that $G_{\text {rest }}$ is not split further, but is still a DAG after $e^{\prime}=\left(u^{\prime}, w\right)$ and $e^{\prime \prime}=\left(u^{\prime}, v\right)$ are added to $E_{\text {rest }}$. We solve the problem by introducing another node $u^{\prime \prime} \neq u^{\prime}$ by setting

$$
V_{\text {rest }}=V_{\text {rest }} \cup\left\{u^{\prime}, u^{\prime \prime}\right\} \quad \text { and } \quad E_{\text {rest }}=E_{\text {rest }} \cup\left\{\left(u^{\prime}, v\right),\left(u^{\prime \prime}, w\right)\right\}
$$

The corresponding export node to $u^{\prime \prime}$ is $u$ in $G\left(v_{1}\right)$. So $v_{1}$ in $G\left(v_{1}\right)$ is the corresponding export node to two import nodes in $G_{\text {rest }}$. $u^{\prime \prime}$ is a permanent import node, because the value of the corresponding export node is still needed to evaluate $v$. If $u=v_{1}$ has not been marked as export node before, then $u^{\prime}$ is a non-permanent import node, because the register containing $u$ can be freed after $v$ is evaluated. One splitting step is executed by the following function split_dag: 
(1) function split_dag(node $v, v_{1}, v_{2}$, dag $\left.G=(V, E)\right)$ : dag;

$/ / v$ is a decision node with children $v_{1}$ and $v_{2} / /$

(2) $u_{1}=$ new_node();

(3) $V=V \cup\left\{u_{1}\right\} ; E=E \cup\left\{\left(u_{1}, v\right)\right\}$;

(4) if $\exp \left(v_{1}\right)==0$ then $i m p_{n p}\left(u_{1}\right)=1$ else $i m p_{p}\left(u_{1}\right)=1 ; \mathbf{f}$;

(5) $\exp \left(v_{1}\right)=1 ; \operatorname{corr}\left(u_{1}\right)=v_{1}$;

(6) delete $\left(v_{1}, v\right)$ from $E$;

(7) for each edge $e=\left(v_{1}, w\right) \in E \mathbf{d o}$

(8) $\quad u_{1}=$ new_node();

(9) $\quad V=V \cup\left\{u_{1}\right\} ; E=E \cup\left\{\left(u_{1}, w\right)\right\}$;

(10) $\quad i m p_{p}\left(u_{1}\right)=1 ; \operatorname{corr}\left(u_{1}\right)=v_{1}$;

(11) delete $e$ from $E$;

(12) od;

(13) Let $G(v)=(V(v), E(v))$ be the subDAG of $G$ with root $v$, let $G\left(v_{1}\right)=\left(V\left(v_{1}\right), E\left(v_{1}\right)\right)$ be the subDAG of $G$ with root $v_{1}$ build $G_{\text {rest }}=\left(V_{\text {rest }}, E_{\text {rest }}\right)$ with $V_{\text {rest }}=V(v)-V\left(v_{1}\right), E_{\text {rest }}=E(v) \cap\left(V_{\text {rest }} \times V_{\text {rest }}\right)$;

(14) for each $u \in V\left(v_{1}\right)$ do

$$
\text { if } \exists w_{1}, \ldots, w_{n} \in V_{\text {rest }} \text { with }\left(u, w_{i}\right) \in E(v)
$$

then $u_{1}=$ new_node();

$$
V=V \cup\left\{u_{1}\right\} ; E=E \cup\left\{\left(u_{1}, w_{i}\right), 1 \leq i \leq n\right\} ;
$$$$
\text { if } \exp (u)==0 \text { then } i m p_{n p}\left(u_{1}\right)=1 \text { else } i m p_{p}\left(u_{1}\right)=1 ; \mathbf{f}
$$$$
\exp (u)=1 ; \operatorname{corr}\left(u_{1}\right)=u \text {; }
$$

delete $\left(u, w_{i}\right)$ from $E, 1 \leq i \leq n$;

(22) od;

(23) Let $G_{r e t}$ be the subDAG of $G$ with root $v$;

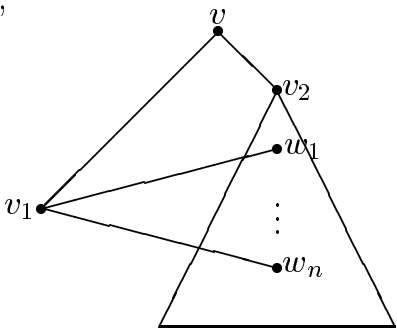

(24) return $G_{r e t}$;

(25) end split_dag;

new_node is a function that returns a new node $x$ and sets $\exp (x), i m p_{p}(x)$ and $i m p_{n p}(x)$ to 0 . split_dag is called by the recursive procedure descend2 that visits the decision nodes in reverse topological order (in the same way as descend). For each decision node $v$ with children $v_{1}$ and $v_{2}$, descend2 executes two possible split operations by using the complete subDAGs with roots $v_{1}$ and $v_{2}$. For each split operation, two subDAGs $G_{\text {left }}$ and $G_{\text {right }}$ are built. If one of these subDAGs is a tree, all decision nodes in the tree are marked with a $*$ so that no further split is executed for these decision nodes. The root of the tree is stored in roots. roots is a set of nodes that is empty at the beginning. If all decision nodes are computed, the trees that have their roots in roots are evaluated according to ord with the modified labeling scheme labelfs2 presented in the next section. 
To evaluate a DAG $G$, we start descend2 $(\Theta, 1, G)$ where $\Theta$ is a bitvector with 0 's at all positions. The decision nodes $v_{1}, \ldots, v_{d}$ are supposed to be sorted in reversed topological order (the root first).

(1) function descend2 ( bitvector $\beta$, int pos, dag $G$ )

(2) while $\beta_{\text {pos }}=*$ and $p o s \leq d$ do $p o s=p o s+1$ od;

(3) if pos $==d+1$

(4) then ord $=$ top_sort $($ roots);

(5) for $i=1$ to $d$ do labelfs2(ord(i)) od;

(6) else $\beta_{\text {pos }}=0 ; G_{1}=\operatorname{copy}(G)$;

(7) mark exclusions of nodes $v_{j}, j \in\{$ pos $+1, \ldots, d\}$ through $l s o n\left(v_{\text {pos }}\right)$ with $\beta_{j}=*$;

(8) $\quad G_{\text {left }}=$ complete subDAG of $G_{1}$ with root $\operatorname{lson}\left(v_{\text {pos }}\right)$

(9) if is_tree $\left(G_{\text {left }}\right)$

(10) then mark all decision nodes in $G_{\text {left }}$ with a $*$; roots $=$ roots $\cup\left\{l \operatorname{son}\left(v_{\text {pos }}\right)\right\} \mathbf{f}$;

(11) $\quad G_{\text {right }}=\operatorname{split} \_\operatorname{dag}\left(v_{\text {pos }}, \operatorname{lson}\left(v_{\text {pos }}\right), \operatorname{rson}\left(v_{\text {pos }}\right), G_{1}\right)$;

(12) if is_tree $\left(G_{\text {right }}\right)$

(13) then mark all decision nodes in $G_{\text {right }}$ with a $*$; roots $=$ roots $\cup\left\{v_{\text {pos }}\right\} \mathbf{f}$;

(14) descend2( $\beta$, pos $\left.+1, G_{1}\right)$;

(15) $\quad \beta_{\text {pos }}=1 ; G_{2}=\operatorname{copy}(G)$;

(16) mark exclusions of nodes $v_{j}, j \in\{$ pos $+1, \ldots, d\}$ through $r s o n\left(v_{p o s}\right)$ with $\beta_{j}=*$;

(17) $\quad G_{\text {right }}=$ complete subDAG of $G_{2}$ with root $\operatorname{rson}\left(v_{\text {pos }}\right)$

(18) if is_tree $\left(G_{\text {right }}\right)$

(19) then mark all decision nodes in $G_{\text {right }}$ with a $*$; roots $=\operatorname{roots} \cup\left\{r \operatorname{rson}\left(v_{\text {pos }}\right)\right\} \mathbf{f}$;

(20) $\quad G_{\text {left }}=\operatorname{split} d a g\left(v_{\text {pos }}, r s o n\left(v_{\text {pos }}\right)\right.$, lson $\left.\left(v_{\text {pos }}\right), G_{2}\right)$;

(21) if is_tree $\left(G_{\text {left }}\right)$

(22) then mark all decision nodes in $G_{\text {left }}$ with a $*$; roots $=\operatorname{roots} \cup\left\{v_{\text {pos }}\right\} \mathbf{f}$;

(23) descend2( $\beta$, pos $\left.+1, G_{2}\right)$;

(24) $\mathbf{f i}$

(25) end descend2;

top_sort is a function that sorts the nodes in its argument set in topological order according to the global DAG. If there are nodes $v, v_{1}, v_{2}, w_{1}, w_{2}$ where $v=v_{\text {pos }}$ is a decision node with $\beta_{\text {pos }}=0$ and $\left(v_{1}, v\right),\left(v_{2}, v\right) \in E$ and $w_{1}$ is a predecessor of $v_{1}$ and $w_{2}$ is a predecessor of $v_{2}$, then $\operatorname{ord}\left(w_{1}\right)<\operatorname{ord}\left(w_{2}\right)$. If $\beta_{\text {pos }}=1$, then $\operatorname{ord}\left(w_{2}\right)<\operatorname{ord}\left(w_{1}\right)$. copy is a function that yields a copy of the argument DAG. is_tree $(G)$ returns true, if $G$ is a tree.

By fixing the evaluation order of the trees, we also determine the type of the import nodes ${ }^{4}$ and thus which import nodes return a free register after their evaluation. An import node is non-permanent

\footnotetext{
${ }^{4}$ If two import nodes $v_{1}$ and $v_{2}$ of the same tree $T_{i}$ have the same corresponding export node, then the type is determined according to the evaluation order of $T_{i}$ as described in the nect section. For the moment we suppose that both nodes are permanent.
} 
if it is the last reference to the corresponding export node. Otherwise it is permanent: The register cannot be freed until the last referencing import node is computed.

\section{$6 \quad$ Evaluating trees with import and export nodes}

We suppose that we have a number of trees $T_{1}=\left(V_{1}, E_{1}\right), \ldots, T_{k}=\left(V_{k}, E_{k}\right)$ with import and export nodes after the split operation executed by descend2. In this section, we describe how an optimal evaluation is generated for these trees. With the definitions from Section 4 we define the following two functions occ and freed:

$$
o c c: V \rightarrow\{0,1\} \quad \text { with } \quad o c c(v)=\sum_{w \text { is a proper predecessor of } v} \exp (w)
$$

counts the number of export nodes in the subtree $T(v)$ with root $v$ (excluding $v$ ), i.e. the number of registers that remain occupied after $T(v)$ has been evaluated.

$$
\text { freed }: V \rightarrow\{0,1\} \quad \text { with } \quad f r e e d(v)=\sum_{w \text { is a proper predecessor of } v} i m p_{n p}(w)
$$

counts the number of import nodes of the second type in $T(v)$, i.e. the number of registers that are freed after $T(v)$ has been evaluated.

We now define for each node $v$ of a tree $T_{i}(1 \leq i \leq k)$ a label label $(v)$ which specifies the number of registers required to evaluate $v$ as follows:

If $v$ is a leaf, then $\operatorname{label}(v)=2-2 \cdot\left(i m p_{p}(v)+i m p_{n p}(v)\right)$. Let $v$ be an inner node with two children $v_{1}$ and $v_{2}$. Let $S_{i}$ be the subtree with root $v_{i}, i=1,2$. We have two possibilities to evaluate $v$, when we use contiguous evaluations: If we evaluate $S_{1}$ before $S_{2}$, we use

$$
m_{1}=\max \left(\operatorname{label}\left(v_{1}\right), \operatorname{label}\left(v_{2}\right)+\operatorname{occ}\left(v_{1}\right)+1-\operatorname{freed}\left(v_{1}\right)\right)
$$

registers, provided that $v_{1}\left(v_{2}\right)$ can be evaluated with label $\left(v_{1}\right)$ (label $\left(v_{2}\right)$ ) registers. After $S_{1}$ is evaluated, we need $\operatorname{occ}\left(v_{1}\right)$ registers to hold the export nodes of $S_{1}$ and one register to hold $v_{1}$. On the other hand, we free freed $\left(v_{1}\right)$ registers, when evaluating $S_{1}$. If we evaluate $S_{2}$ before $S_{1}$, we use

$$
m_{2}=\max \left(\operatorname{label}\left(v_{2}\right), \operatorname{label}\left(v_{1}\right)+\operatorname{occ}\left(v_{2}\right)+1-\operatorname{freed}\left(v_{2}\right)\right)
$$

registers. We suppose that the best evaluation order is chosen and set

$$
\operatorname{label}(v)=\min \left(m_{1}, m_{2}\right)
$$

The following algorithm generates an evaluation for a labeled tree $T$ with root $v$ :

(1) function labelfs2 ( node $v$ )

(2) if $v$ is a leaf

(3) then if $i m p_{p}(v)+i m p_{n p}(v)==0$ then $\operatorname{reg}(v)=n e w \_r e g()$; print $(v, r e g(v)) \mathbf{f i}$

(4) else if $v$ is an inner node with $l \operatorname{son}(v)=v_{1}$ and $r \operatorname{son}(v)=v_{2}$ 


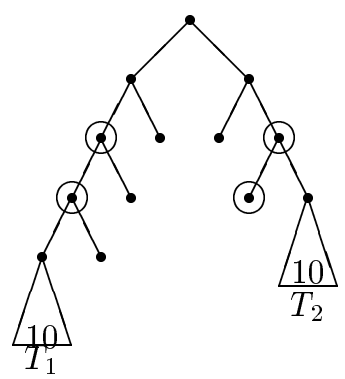

Figure 9: $T_{1}$ and $T_{2}$ are complete binary trees with height 9, so we need 10 registers to evaluate each of them. The export nodes are marked with a circle. If a contiguous evaluation is used, we must first evaluate the left or the right subtree of the root completely before starting the evaluation of the other subtree. We need at least 13 registers, because 3 registers are required to hold the export nodes and the root node of the other subtree. A non-contiguous evaluation can evaluate the tree with 11 registers by first evaluating $T_{1}$, then $T_{2}$ and then the rest of the tree.
(12) fi;

$$
\begin{aligned}
& \text { then if } \operatorname{label}\left(v_{1}\right)+\operatorname{occ}\left(v_{2}\right)-f r e e d\left(v_{2}\right) \geq \operatorname{label}\left(v_{2}\right)+\operatorname{occ}\left(v_{1}\right)-f r e e d\left(v_{1}\right) \\
& \quad \text { then labelfs } 2\left(v_{1}\right) ; \operatorname{labelfs} 2\left(v_{2}\right) ; \\
& \text { else labelfs } 2\left(v_{2}\right) ; \operatorname{labelfs} 2\left(v_{1}\right) ; \\
& \quad \mathbf{f} \\
& \quad \operatorname{reg}(v)=\text { new_reg }() ; \operatorname{print}(v, \operatorname{reg}(v)) ; \mathbf{f i} \\
& \text { if } \exp \left(v_{1}\right)==0 \text { then } \operatorname{regfree}\left(\operatorname{reg}\left(v_{1}\right)\right) ; \mathbf{f i} \\
& \text { if } \exp \left(v_{2}\right)==0 \text { then } \operatorname{regfree}\left(\operatorname{reg}\left(v_{2}\right)\right) ; \mathbf{f i}
\end{aligned}
$$

Now we will prove that the call labelfs2( $v$ ) generates an optimal contiguous evaluation of $v$ and uses label $(v)$ registers. We prove this by two lemmata:

Lemma 7 Let $T=(V, E)$ be a tree and $v \in V$ be an arbitrary inner node of $T$. labelfs2 generates an evaluation for $v$ that uses label $(v)$ registers.

Lemma 8 Let $T=(V, E)$ be a tree and $v \in V$ be an arbitrary inner node of $T$. label( $v)$ is a lower bound for the minimal number of registers needed by a contiguous evaluation for $v$.

Lemma 7 and Lemma 8 result in the following theorem:

Theorem 9 The presented algorithm generates a contiguous evaluation that uses no more registers than any other contiguous evaluation.

However, there may be a non-contiguous evaluation that needs fewer registers than the generated contiguous one. An example is given in Figure 9.

Until now, we have assumed that two different import nodes of a tree $T_{i}$ have different corresponding export nodes. We now explain what has to be done if this is not true. Let $A=\left\{w_{1}, \ldots, w_{n}\right\} \subseteq V_{i}$ be a set of import nodes of $T_{i}$ with the same corresponding export node that is stored in a register $r$. As described above we have set

$$
i m p_{p}\left(w_{1}\right)=\ldots=i m p_{p}\left(w_{n}\right)=1 \text { and } i m p_{n p}\left(w_{1}\right)=\ldots=i m p_{n p}\left(w_{n}\right)=0
$$


But $r$ can be freed, after the last node of $A$ is evaluated. By choosing an appropriate node $w \in A$ to be evaluated last, $T_{i}$ eventually can be evaluated with one register less than the label of the root specifies. We determine $w$ by a top-down traversal of $T_{i}$. Let $v$ be an inner node of $T_{i}$ with children $v_{1}$ and $v_{2}$. Let $S_{j}$ be the subtree with root $v_{j}, j=1,2$. If only one of $S_{1}$ and $S_{2}$ contains nodes of A, we descend to the root of this tree. If both $S_{1}$ and $S_{2}$ contain nodes of A, we examine, whether we can decrease the label value of $v$ by choosing $S_{1}$ or $S_{2}$. Let be $a=\operatorname{label}\left(v_{1}\right)+\operatorname{occ}\left(v_{2}\right)-\operatorname{freed}\left(v_{2}\right)$ and $b=$ label $\left(v_{2}\right)+\operatorname{occ}\left(v_{1}\right)-\operatorname{freed}\left(v_{1}\right)$ If $a>b$, this can only be achieved by searching $w$ in $S_{1}$. If $a<b$, this can only be achieved by searching $w$ in $S_{2}$. If $a=b$, we cannot decrease the register need and can search in $S_{1}$ or $S_{2}$.

We repeat this process until we reach a leaf $w \in A$. We set $i m p_{p}(w)=0, i m p_{n p}(w)=1$.

\section{Experimental Results}

We have implemented descend and descend2 and have applied them to a great variety of randomly generated test DAGs with up to 150 nodes and to large DAGs taken from real application programs, see Tables 2 and 3. The random DAGs are generated by initializing a predefined number of nodes and by selecting a certain number of leaf nodes. Then, the children of inner nodes are selected randomly. The following observations can be made:

- descend reduces the number of different contiguous evaluations considerably.

- descend2 often leads to a large additional improvement over descend, especially for DAGs where descend is not so successful in reducing the number of different contiguous evaluations.

- descend2 works even better for DAGs from real application programs than for random DAGs.

- Only one of the considered DAGs with $n \leq 25$ nodes has a non-contiguous evaluation that uses fewer registers than the computed contiguous evaluation. ${ }^{5}$

- In almost all cases, the computational effort of descend2 seems to be justified. This means that, in practice, an optimal contiguous evaluation (and thus, contiguous register allocation) can be computed in acceptable time even for large DAGs.

\section{Register Allocation}

After the evaluation order is determined, we can compute the register allocation.

\footnotetext{
${ }^{5}$ For a subDAG of MDG with $n=24$ nodes, there is a non-contiguous that uses 6 registers. The computed contiguous evaluation takes 7 registers. The program to compute the non-contiguous evaluation has run for about 7 days, the corresponding program for the contiguous evaluation took less than 0.1 seconds. For DAGs with $n>25$ nodes it is not possible to compute the best non-contiguous evaluation because of the runtime of the program that computes them is growing too fast.
} 


\begin{tabular}{|c|c||c|c|c|}
\hline$n$ & $d$ & $N_{\text {simple }}$ & $N_{\text {descend }}$ & $N_{\text {descend } 2}$ \\
\hline \hline 24 & 12 & 4096 & 146 & 5 \\
25 & 14 & 16384 & 1248 & 3 \\
28 & 16 & 65536 & 748 & 22 \\
27 & 17 & 131072 & 744 & 15 \\
28 & 19 & 524288 & 630 & 32 \\
33 & 21 & 2097152 & 1148 & 98 \\
36 & 24 & 16777216 & 2677 & 312 \\
38 & 26 & 67108864 & 6128 & 408 \\
39 & 27 & 134217728 & 1280 & 358 \\
42 & 29 & 536870912 & 6072 & 64 \\
42 & 31 & $2^{31}$ & 2454 & 152 \\
46 & 34 & $2^{34}$ & 4902 & 707 \\
54 & 39 & $2^{39}$ & 30456 & 592 \\
56 & 43 & $2^{43}$ & 21048 & 4421 \\
\hline
\end{tabular}

\begin{tabular}{|c|c||c|c|c|}
\hline$n$ & $d$ & $N_{\text {simple }}$ & $N_{\text {descend }}$ & $N_{\text {descend } 2}$ \\
\hline \hline 20 & 14 & 16384 & 160 & 10 \\
28 & 16 & 65536 & 784 & 8 \\
29 & 18 & 262144 & 938 & 32 \\
30 & 21 & 2097152 & 1040 & 64 \\
37 & 23 & 8388608 & 13072 & 24 \\
38 & 24 & 16777216 & 11924 & 56 \\
45 & 27 & 134217728 & 100800 & 18 \\
41 & 29 & 536870912 & 74016 & 364 \\
41 & 31 & $2^{31}$ & 3032 & 142 \\
41 & 31 & $2^{31}$ & 3128 & 180 \\
44 & 33 & $2^{33}$ & 40288 & 435 \\
46 & 34 & $2^{34}$ & 40244 & 1008 \\
48 & 37 & $2^{37}$ & 21488 & 1508 \\
53 & 42 & $2^{42}$ & 79872 & 3576 \\
\hline
\end{tabular}

Table 2: Some examples from a test series for large random DAGs. The number of contiguous evaluations generated by the algorithms simple, descend and descend2 are given for typical examples. The tests confirm the large improvements of descend and descend2.

\begin{tabular}{|c|c|c|c||c|c|c|c|c|}
\hline Source & DAG & $n$ & $d$ & $N_{\text {simple }}$ & $N_{\text {descend }}$ & $N_{\text {descend } 2}$ & $T_{\text {descend }}$ & $T_{\text {descend } 2}$ \\
\hline \hline LL 14 & second loop & 19 & 10 & 1024 & 432 & 18 & 0.1 sec. & $<0.1$ sec. \\
\hline LL 20 & inner loop & 23 & 14 & 16384 & 992 & 6 & $0.2 \mathrm{sec}$. & $<0.1$ sec. \\
\hline MDG & calc. $\cos (\theta), \sin (\theta), \ldots$ & 26 & 15 & 32768 & 192 & 96 & $<0.1$ sec. & $<0.1$ sec. \\
\hline MDG & calc. forces, first part & 81 & 59 & $2^{59}$ & - & 7168 & - & 13.6 sec. \\
& subDAG of this & 65 & 45 & $2^{45}$ & - & 532 & - & 0.9 sec. \\
& subDAG of this & 52 & 35 & $2^{35}$ & 284672 & 272 & $70.2 \mathrm{sec}$. & 0.8 sec. \\
& subDAG of this & 44 & 30 & $2^{30}$ & 172032 & 72 & 42.9 sec. & 0.3 sec. \\
& subDAG of this & 24 & 12 & 4096 & 105 & 8 & $<0.1 \mathrm{sec}$. & $<0.1$ sec. \\
\hline SPEC77 & mult. FFT analysis & 49 & 30 & $2^{30}$ & 131072 & 32768 & $20.05 \mathrm{sec}$. & $21.1 \mathrm{sec}$. \\
\hline
\end{tabular}

Table 3: Some measurements for DAGs taken from real programs (LL = Livermore Loop Kernels; MDG = Molecular Dynamics, and SPEC77 = atmospheric flow simulation, both from the Perfect Club Benchmark Suite). The table also gives the run times of the algorithms descend and descend2, implemented on a SUN SPARC station SLC. The tests show that for large DAGs descend is too slow. but the run times required by descend2 remain really acceptable. 
Our experiments have shown that the reordering of large basic blocks according to an optimal contiguous evaluation saves about $30 \%$ of the required registers on the average (see [11]).

We use a register allocation scheme called first_free_reg that allocates, for each node, the free register with the smallest number. Since a new register is allocated only if there is no other free register left, the generated register allocation is optimal and the number of allocated registers is equal to the register need of the evaluation.

The register allocation scheme uses a binary tree with the register $1, \ldots, n$ as leaves. In each node, there is a flag free that indicates, whether the subtree of this node contains a free register. In order to allocate a free register, we walk along a path from the root to a free register by turning at each node to its leftmost son with a TRUE free flag. After switching the flag of the leaf found to FALSE, we traverse the path back to the root in order to update the flags. For each node on the path we set free to FALSE iff its two sons have free = FALSE.

If a register is marked free again, we must restore the free flags on the path from this register back to the root in the same way by setting for each node free to TRUE if at least one son has a true free flag. The run time is $O(\log n)$ for allocating or freeing a register, thus the total run time is $O(n \log n)$ for the evaluation of a DAG with $n$ nodes.

The advantage of this allocation method is that the allocated registers usually have rather different access rates since, in general, registers with a low number are used more often than registers with a high number. That results in an allocation scheme that is well suited for spilling registers. If we have fewer registers available in the target machine than the evaluation requires, then we are forced to spill those registers with the least usage. The spill cost are at a minimum, if usage is distributed as unequally as possible over the allocated registers. The proposed heuristic first_free_reg fulfills this condition quite well. The actual spilling algorithm is described in [2] for basic blocks of vector instructions and may easily be adapted for the scalar case.

The general problem of computing an evaluation that is optimal with respect to spill cost seems to be a hard problem in terms of computational complexity, but that does not really matter in practice because a possible further gain in execution time compared to first_free_reg appears to be marginal for real DAGs.

\section{Conclusions}

We have presented two variants of the simple algorithm that evaluates only the tree nodes by a labeling algorithm and generates $2^{d}$ contiguous evaluations where $d$ is the number of decision nodes of the DAG. The first variant is the exclusion of redundant decision nodes as performed by procedure descend. The second variant is the splitting of the DAG in subtrees (performed by descend2) and the evaluation of these by the modified labeling algorithm labelfs2. The experimental results in Section 7 confirm that this variant generates only a small number of contiguous evaluations, even for large DAGs. Among the generated evaluations are all evaluations with the least register need. Therefore, 
by using descend2 we find the optimal contiguous evaluation in a reasonable time even for large DAGs. The dramatic reduction in evaluations generated makes descend2 suitable for the use in optimizing compilers, especially for time-critical regions of the source program.

\section{Acknowledgements}

The authors would like to thank Prof. Dr. R. Wilhelm and Prof. Dr. W.J. Paul for their helpful support.

\section{References}

[1] The Connection Machine CM-5 Technical Summary, Thinking Machines Corporation, Cambridge, MA, 1991

[2] Keßler, C.W., Paul, W.J., Rauber, T.: Scheduling Vector Straight Line Code on Vector Processors. in: R. Giegerich, S.L. Graham (Ed.): Code Generation - Concepts, Tools, Techniques. Springer Workshops in Computing Series (WICS), 1992.

[3] Dongarra, J.J., Jinds, A.R.: Unrolling Loops in Fortran, Software Practice and Experience, 9:3, 219-226 (1979)

[4] Fisher, J,: Trace Scheduling: A Technique for Global Microcode Compaction, IEEE Transactions on Computers, C-30:7 (1981)

[5] Goodman J.R., Hsu Wei-Chung: Code Scheduling and Register Allocation in Large Basic Blocks, ACM International Conference on Supercomputing, 1988, 442-452

[6] Chaitin, G.J., Auslander M.A., Chandra A.K., Cocke J., Hopkins M.E., Markstein P.W.: Register allocation via coloring. Computer Languages Vol. 6, 47-57 (1981)

[7] Chaitin, G.J.: Register allocation \& spilling via graph coloring. ACM SIGPLAN Notices 17:6, 201-207 (1982)

[8] Aho, A.V., Sethi, R., Ullman, J.D.: Compilers: Principles, Techniques, and Tools. Addison-Wesley (1986)

[9] Sethi, R., Ullman, J.D.: The generation of optimal code for arithmetic expressions. Journal of the ACM, Vol. 17, 715-728 (1970)

[10] Sethi, R.: Complete register allocation problems. SIAM Journal of Computing 4, 226-248 (1975)

[11] Keßler, C.W., Paul, W.J., Rauber, T.: A Randomized Heuristic Approach to Register Allocation. Proceedings of PLILP'91 Third International Symposium on Programming Language Implementation and Logic Programming, Aug. 26-28, 1991, Passau, Germany. Springer LNCS Vol. 528, 195-206.

[12] Mehlhorn, K.: Data Structures and Algorithms 2: Graph Algorithms and NP-Completeness. (1984)

[13] Aho A.V., Johnson S.C.: Optimal Code Generation for Expression Trees, Journal of the ACM 23:3 (1976), pages 488-501

[14] Rauber, Thomas: An Optimizing Compiler for Vector Processors. Proc. ISMM International Conference on Parallel and Distributed Computing and Systems, New York 1990, Acta press, 97-103 